\title{
On analogues of black brane solutions in the model with multicomponent anisotropic fluid
}

\author{
V. D. Ivashchuk ${ }^{1}$, \\ Center for Gravitation and Fundamental Metrology, VNIIMS, 46 Ozyornaya ul., Moscow 119361, Russia \\ Institute of Gravitation and Cosmology, Peoples' Friendship University of Russia, 6 Miklukho-Maklaya ul., \\ Moscow 117198, Russia
}

\begin{abstract}
A family of spherically symmetric solutions with horizon in the model with $m$-component anisotropic fluid is presented. The metrics are defined on a manifold that contains a product of $n-1$ Ricci-flat "internal" spaces. The equation of state for any $s$-th component is defined by a vector $U^{s}$ belonging to $\mathbb{R}^{n+1}$. The solutions are governed by moduli functions $H_{s}$ obeying non-linear differential equations with certain boundary conditions imposed. A simulation of black brane solutions in the model with antisymmetric forms is considered. An example of solution imitating $M_{2}-M_{5}$ configuration (in $D=11$ supergravity) corresponding to Lie algebra $A_{2}$ is presented.
\end{abstract}

\section{Introduction}

In this paper we continue our investigations of spherically-symmetric solutions with horizon (e.g., black brain ones) defined on product manifolds containing several Ricci-flat factor-spaces (with diverse signatures and dimensions). These solutions appear either in models with antisymmetric forms and scalar fields [1-[11] or in models with (multicomponent) anisotropic fluid [12]-[15]. For black brane solutions with 1-dimensional factor-spaces (of Euclidean signatures) see [16, 17, 18, and references therein.

These and more general brane cosmological and spherically symmetric solutions were obtained by reduction of the field equations to the Lagrange equations corresponding to Toda-like systems [2, 19]. An analogous reduction for models with multicomponent anisotropic fluids was performed earlier in [20, 21]. For cosmological-type models with antisymmetric forms without scalar fields any brane is equivalent to an anisotropic fluid with the equations of state:

$$
\hat{p}_{i}=-\hat{\rho} \quad \text { or } \quad \hat{p}_{i}=\hat{\rho},
$$

when the manifold $M_{i}$ belongs or does not belong to the brane world volume, respectively (here $\hat{p}_{i}$ is the effective pressure in $M_{i}$ and $\hat{\rho}$ is the effective density).

In this paper we present spherically-symmetric solutions with horizon (e.g the analogues of intersecting black brane solutions) in a model with multi-component anisotropic fluid (MCAF), when certain relations on fluid parameters are imposed. The solutions are governed by a set of moduli functions $H_{s}$ obeying non-linear differential master equations with certain boundary conditions imposed. These master equations are equaivalent to Toda-like equations and depend upon the non-degenerate $(m \times m)$ matrix $A$. It was conjectured earlier that the functions $H_{s}$ should be polynomials when $A$ is a Cartan matrix for some semi-simple finite-dimensional Lie algebra (of rank $m$ ) [6. This conjecture was verified for Lie algebras: $A_{m}, C_{m+1}, m \geq 1$ [7, 8. A special case of black hole solutions with MCAF corresponding to semisimple Lie algebra $A_{1} \oplus \ldots \oplus A_{1}$ was considered earlier in [13] (for $m=1$ see [12]).

The paper is organized as follows. In Section 2 the model is formulated. In Section 3 spherically-symmetric MCAF solutions with horizon corresponding to black-brane-type solutions, are presented. In Section 4 a polynomial structure of moduli functions $H_{s}$ for semi-simple finite-dimensional Lie algebras is discussed. In Section 5 a simulation of intersecting black brane solutions is considered and an analogue of $M 2-M 5$ dyonic solution is presented.

\section{The model}

In this paper we deal with a family of spherically symmetric solutions to Einstein equations with an anisotropic matter source

$$
R_{N}^{M}-\frac{1}{2} \delta_{N}^{M} R=k^{2} T_{N}^{M},
$$

\footnotetext{
${ }^{1}$ e-mail: ivashchuk@mail.ru
} 
defined on the manifold

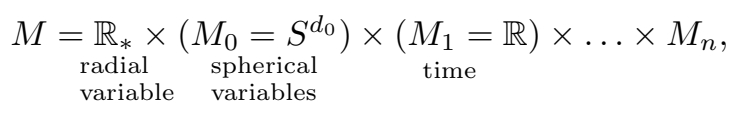

with the block-diagonal metrics

$$
d s^{2}=\mathrm{e}^{2 \gamma(u)} d u^{2}+\sum_{i=0}^{n} \mathrm{e}^{2 \beta^{i}(u)} h_{m_{i} n_{i}}^{[i]} d y^{m_{i}} d y^{n_{i}} .
$$

Here $\mathbb{R}_{*} \subseteq \mathbb{R}$ is an open interval. The manifold $M_{i}$ with the metric $h^{[i]}, i=1,2, \ldots, n$, is a Ricci-flat space of dimension $d_{i}$ :

$$
R_{m_{i} n_{i}}\left[h^{[i]}\right]=0,
$$

and $h^{[0]}$ is the standard metric on the unit sphere $S^{d_{0}}$, so that

$$
R_{m_{0} n_{0}}\left[h^{[0]}\right]=\left(d_{0}-1\right) h_{m_{0} n_{0}}^{[0]}
$$

$u$ is a radial variable, $\kappa^{2}$ is the gravitational constant, $d_{1}=1$ and $h^{[1]}=-d t \otimes d t$.

The energy-momentum tensor is adopted in the following form for each component of the fluid:

$$
\left(T_{N}^{s M}\right)=\operatorname{diag}\left(-\hat{\rho}^{s}, \hat{p}_{0}^{s} \delta_{k_{0}}^{m_{0}}, \hat{p}_{1}^{s} \delta_{k_{1}}^{m_{1}}, \ldots, \hat{p}_{n}^{s} \delta_{k_{n}}^{m_{n}}\right),
$$

where $\hat{\rho}^{s}$ and $\hat{p}_{i}^{s}$ are the effective density and pressures respectively, depending on the radial variable $u$.

We assume that the following "conservation laws"

$$
\nabla_{M} T_{N}^{(s) M}=0
$$

are valid for all components.

We also impose the following equations of state

$$
\hat{p}_{i}^{s}=\left(1-\frac{2 U_{i}^{s}}{d_{i}}\right) \hat{\rho}^{s}
$$

where $U_{i}^{s}$ are constants, $i=0,1, \ldots, n$.

The physical density and pressures are related to the effective ones (with "hats") by the formulae

$$
\rho^{s}=-\hat{p}_{1}^{s}, \quad p_{u}^{s}=-\hat{\rho}^{s}, \quad p_{i}^{s}=\hat{p}_{i}^{s} \quad(i \neq 1) .
$$

In what follows we put $\kappa=1$ for simplicity.

\section{Spherically symmetric solutions with horizon}

We will make the following assumptions:

$$
\begin{array}{ll}
1^{o} . & U_{0}^{s}=0 \Leftrightarrow \hat{p}_{0}^{s}=\hat{\rho}^{s}, \\
2^{o} . & U_{1}^{s}=1 \Leftrightarrow \hat{p}_{1}^{s}=-\hat{\rho}^{s}, \\
3^{o} . & \left(U^{s}, U^{s}\right)=U_{i}^{s} G^{i j} U_{j}^{s}>0, \\
4^{o} . & 2\left(U^{s}, U^{l}\right) /\left(U^{l}, U^{l}\right)=A_{s l},
\end{array}
$$

where $A=\left(A_{s l}\right)$ is non-degenerate matrix,

$$
G^{i j}=\frac{\delta^{i j}}{d_{i}}+\frac{1}{2-D}
$$

are components of the matrix inverse to the matrix of the minisuperspace metric 22$]$

$$
\begin{aligned}
& \left(G_{i j}\right)=\left(d_{i} \delta_{i j}-d_{i} d_{j}\right) \\
& i, j=0,1, \ldots, n \text { and } D=1+\sum_{i=0}^{n} d_{i} \text { is the total dimension. }
\end{aligned}
$$


The conditions $1^{\circ}$ and $2^{\circ}$ in brane terms mean that brane "lives" in the time manifold $M_{1}$ and does not "live" in $M_{0}$. Due to assumptions $1^{\circ}$ and $2^{\circ}$ and the equations of state (2.8), the energy-momentum tensor (2.6) reads as follows:

$$
\left(T_{N}^{(s)}{ }_{N}^{M}\right)=\operatorname{diag}\left(-\rho^{s}, \rho^{s} \delta_{k_{0}}^{m_{0}},-\rho^{s}, p_{2}^{s} \delta_{k_{2}}^{m_{2}}, \ldots, p_{n}^{s} \delta_{k_{n}}^{m_{n}}\right)
$$

Under the conditions (2.8) and (3.1) we have obtained the following black-hole solutions to the Einstein equations (2.1):

$$
\begin{aligned}
& d s^{2}=J_{0}\left(\frac{d r^{2}}{1-2 \mu / R^{d}}+R^{2} d \Omega_{d_{0}}^{2}\right)-J_{1}\left(1-\frac{2 \mu}{R^{d}}\right) d t^{2}+\sum_{i=2}^{n} J_{i} h_{m_{i} n_{i}}^{[i]} d y^{m_{i}} d y^{n_{i}}, \\
& \rho^{(s)}=-\frac{A_{s}}{J_{0} R^{2 d_{0}}} \prod_{l=1}^{m} H_{l}^{-A_{s l}}
\end{aligned}
$$

which may be derived by analogy with the black brane solutions [7, 8]. Here $d=d_{0}-1$,

$$
d \Omega_{d_{0}}^{2}=h_{m_{0} n_{0}}^{[0]} d y^{m_{0}} d y^{n_{0}}
$$

is the $d_{0}$-dimensional spherical element (corresponding to the metric on $S^{d_{0}}$ ),

$$
J_{i}=\prod_{s=1}^{m} H_{s}^{-2 h_{s} U^{s i}}
$$

$i=0,1, \ldots, n, \mu>0$ is integration constant and

$$
\begin{aligned}
& U^{s i}=G^{i j} U_{j}^{s}=\frac{U_{i}^{s}}{d_{i}}+\frac{1}{2-D} \sum_{j=0}^{n} U_{j}^{s}, \\
& h_{s}=K_{s}^{-1}, \quad K_{s}=\left(U^{s}, U^{s}\right) .
\end{aligned}
$$

It follows from $1^{\circ}$ and (3.9) that

$$
U^{s 0}=\frac{1}{2-D} \sum_{j=1}^{n} U_{j}^{s}
$$

Functions $H_{s}>0$ obey the equations

$$
R^{d_{0}} \frac{d}{d R}\left[\left(1-\frac{2 \mu}{R^{d}}\right) \frac{R^{d_{0}}}{H_{s}} \frac{d H_{s}}{d R}\right]=B_{s} \prod_{l=1}^{m} H_{l}^{-A_{s l}},
$$

with $B_{s}=2 K_{s} A_{s}$ and the boundary conditions imposed:

$$
H_{s} \rightarrow H_{s 0} \neq 0, \quad \text { for } \quad R^{d} \rightarrow 2 \mu,
$$

and

$$
H_{s}(R=+\infty)=1
$$

$s=1, \ldots, m$, i.e. the metric (3.5) has a regular horizon at $R^{d}=2 \mu$ and has an asymptotically flat $\left(2+d_{0}\right)-$ dimensional section.

Due to to (3.1) and (3.9) the metric reads

$$
d s^{2}=J_{0}\left[\frac{d r^{2}}{1-2 \mu / R^{d}}+R^{2} d \Omega_{d_{0}}^{2}-\left(\prod_{s=1}^{m} H_{s}^{-2 h_{s}}\right)\left(1-\frac{2 \mu}{R^{d}}\right) d t^{2}+\sum_{i=2}^{n} Y_{i} h_{m_{i} n_{i}}^{[i]} d y^{m_{i}} d y^{n_{i}}\right]
$$

where

$$
Y_{i}=\prod_{s=1}^{m} H_{s}^{-2 h_{s} U_{i}^{s} / d_{i}} .
$$

The solution (3.5), (3.6) may be verified just by a straightforward substitution into equations of motion. A detailed derivation of this solution will be given in a separate paper [26]. A special orthogonal case when $\left(U^{s}, U^{l}\right)=0$, for $s \neq l$, was considered earlier in [13] (for $m=1$ see [12]) More general solutions in orthogonal case (with more general condition instead of $2^{\circ}$ ) were obtained in [15] (for $m=1$ see [14].) 


\section{Polynomial structure of $H_{s}$ for Lie algebras}

Now we deal with solutions to second order non-linear differential equations (3.12) that may be rewritten as follows

$$
\frac{d}{d z}\left(\frac{(1-2 \mu z)}{H_{s}} \frac{d}{d z} H_{s}\right)=\bar{B}_{s} \prod_{l=1}^{m} H_{l}^{-A_{s l}}
$$

where $H_{s}(z)>0, z=R^{-d} \in\left(0,(2 \mu)^{-1}\right)(\mu>0)$ and $\bar{B}_{s}=B_{s} / d^{2} \neq 0$. Eqs. (3.13) and (3.14) read

$$
\begin{array}{r}
H_{s}\left((2 \mu)^{-1}-0\right)=H_{s 0} \in(0,+\infty), \\
H_{s}(+0)=1,
\end{array}
$$

$s=1, \ldots, m$.

It was conjectured in [6] that equations (4.1)-(4.3) have polynomial solutions when $\left(A_{s s^{\prime}}\right)$ is a Cartan matrix for some semisimple finite-dimensional Lie algebra $\mathcal{G}$ of rank $m$. In this case we get

$$
H_{s}(z)=1+\sum_{k=1}^{n_{s}} P_{s}^{(k)} z^{k}
$$

where $P_{s}^{(k)}$ are constants, $k=1, \ldots, n_{s} ; P_{s}^{\left(n_{s}\right)} \neq 0$, and

$$
n_{s}=b_{s} \equiv 2 \sum_{l=1}^{m} A^{s l}
$$

$s=1, \ldots, m$, are the components of twice the dual Weyl vector in the basis of simple co-roots [24]. Here $\left(A^{s l}\right)=$ $\left(A_{s l}\right)^{-1}$.

This conjecture was verified for $\mathbf{A}_{\mathbf{m}}$ and $\mathbf{C}_{\mathbf{m}+\mathbf{1}}$ series of Lie algebras in $[7,8$. In extremal case $(\mu=+0)$ an a analogue of this conjecture was suggested (implicitly) in [25.

$\mathbf{A}_{\mathbf{1}} \oplus \ldots \oplus \mathbf{A}_{1}$-case.

The simplest example occurs in orthogonal case : $\left(U^{s}, U^{l}\right)=0$, for $s \neq l$ [1, 2] (see also [16, 17, 18, and refs. therein). In this case $\left(A_{s l}\right)=\operatorname{diag}(2, \ldots, 2)$ is a Cartan matrix for semisimple Lie algebra $\mathbf{A}_{\mathbf{1}} \oplus \ldots \oplus \mathbf{A}_{\mathbf{1}}$ and

$$
H_{s}(z)=1+P_{s} z
$$

with $P_{s} \neq 0$, satisfying

$$
P_{s}\left(P_{s}+2 \mu\right)=-\bar{B}_{s}=-2 K_{s} A_{s} / d^{2},
$$

$s=1, \ldots, m$. When all $A_{s}<0$ (or, equivalently, $\rho^{s}>0$ ) there exists a unique set of numbers $P_{s}>0$ obeying (4.7).

$A_{2}$-case.

For the Lie algebra $\mathcal{G}$ coinciding with $\mathbf{A}_{\mathbf{2}}=\operatorname{sl}(3)$ we get $n_{1}=n_{2}=2$ and

$$
H_{s}=1+P_{s} z+P_{s}^{(2)} z^{2}
$$

where $P_{s}=P_{s}^{(1)}$ and $P_{s}^{(2)} \neq 0$ are constants, $s=1,2$.

It was found in [6] that for $P_{1}+P_{2}+4 \mu \neq 0$ (e.g. when all $P_{s}>0$ ) the following relations take place

$$
P_{s}^{(2)}=\frac{P_{s} P_{s+1}\left(P_{s}+2 \mu\right)}{2\left(P_{1}+P_{2}+4 \mu\right)}, \quad \bar{B}_{s}=-\frac{P_{s}\left(P_{s}+2 \mu\right)\left(P_{s}+4 \mu\right)}{P_{1}+P_{2}+4 \mu},
$$

$s=1,2$.

Here we denote $s+1=2,1$ for $s=1,2$, respectively.

Other solutions.

At the moment the "master" equations were integrated (using the Maple) in 9, 10] for Lie algebras $\mathbf{C}_{\mathbf{2}}$ and $\mathbf{A}_{\mathbf{3}}$, respectively.

Special solutions $H_{s}(z)=\left(1+P_{s} z\right)^{b_{s}}$ with $b_{s}$ from (4.5) appeared earlier in [3, 4, 5] in a context of so-called block-orthogonal configurations. 


\section{Examples}

\subsection{Simulation of intersecting black branes}

The solution from the previous section for MCAF allows to simulate the intersecting black brane solutions 11 in the model with antisymmetric forms without scalar fields. In this case the parameters $U_{i}^{s}$ and pressures have the following form:

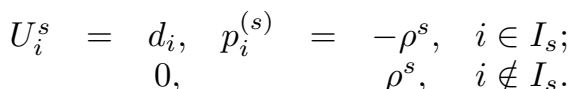

Here $I_{s}=\left\{i_{1}^{s}, \ldots, i_{k_{s}}^{s}\right\} \in\{1, \ldots n\}$ is the index set [1] corresponding to brane submanifold $M_{i_{1}^{s}} \times \ldots \times M_{i_{k_{s}}}$.

The relation $4^{o}$ (3.1) leads us to the following dimensions of intersections of brane submanifolds ("worldvolumes") [2, 11:

$$
\begin{aligned}
& d\left(I_{s} \cap I_{l}\right)=\frac{d\left(I_{s}\right) d\left(I_{l}\right)}{D-2}+\frac{1}{2} K_{l} A_{s l}, \\
& s \neq l ; s, l=1, \ldots, m . \text { Here } d\left(I_{s}\right) \text { and } d\left(I_{l}\right) \text { are dimensions of brane world-volumes. }
\end{aligned}
$$

\section{2 $\quad M_{2}-M_{5}$-analogue for Lie algebra $A_{2}$}

In 13. examples of simulation by MCAF of $M 2 \cap M 5, M 2 \cap M 2, M 5 \cap M 5$ black brane solutions in $D=11$ supergravity, with the standard (orthogonal) intersection rules were considered.

Now we consider a solution with 2-component anisotropic fluid that simulates $M_{2}-M_{5}$ dyonic configuration in $D=11$ supergravity [6], corresponding to Lie algebra $\mathbf{A}_{\mathbf{2}}$.

The solution is defined on the manifold

$$
M=(2 \mu,+\infty) \times\left(M_{0}=S^{2}\right) \times\left(M_{1}=\mathbb{R}\right) \times M_{2} \times M_{3},
$$

where $\operatorname{dim} M_{2}=2$ and $\operatorname{dim} M_{3}=5$. The $U^{s}$-vectors corresponding to fluid components obey (5.1) with $I_{1}=\{1,2\}$ and $I_{2}=\{1,3\}$.

The solution reads as following

$$
\begin{array}{r}
g=H_{1}^{1 / 3} H_{2}^{2 / 3}\left\{\frac{d R \otimes d R}{1-2 \mu / R}+R^{2} d \Omega_{2}^{2}\right. \\
\left.-H_{1}^{-1} H_{2}^{-1}\left(1-\frac{2 \mu}{R}\right) d t \otimes d t+H_{1}^{-1} h^{[2]}+H_{2}^{-1} h^{[3]}\right\}, \\
\rho^{(1)}=-\frac{A_{1}}{J_{0} R^{4}} H_{1}^{-2} H_{2}, \quad \rho^{(2)}=-\frac{A_{2}}{J_{0} R^{4}} H_{1} H_{2}^{-2},
\end{array}
$$

where $J_{0}=H_{1}^{1 / 3} H_{2}^{2 / 3} ; h^{[2]}$ and $h^{[3]}$ are Ricci-flat metrics of Euclidean signatures, $\mu>0$ and $H_{s}$ are defined by (4.8), where $z=R^{-1}$ and parameters $P_{s}, P_{s}^{(2)}, \bar{B}_{s}=B_{s}=4 A_{s}(s=1,2)$ obey (4.9).

This solution simulates $\mathbf{A}_{\mathbf{2}}$-dyon from [6] consisting of electric $M 2$-brane with worldvolume isomorphic to $\left(M_{1}=\mathbb{R}\right) \times M_{2}$ and magnetic $M 5$-brane with worldvolume isomorphic to $\left(M_{1}=\mathbb{R}\right) \times M_{3}$. The branes are intersecting on the time manifold $M_{1}=\mathbb{R}$. Here $K_{s}=\left(U^{s}, U^{s}\right)=2$, for all $s=1,2$.

For $\mathbf{A}_{\mathbf{2}}$-dyon from [6] we had $\bar{B}_{s}=B_{s}=-2 Q_{s}^{2}$, where $Q_{s}$ is the charge density parameter of $s$-th brane. Thus, for fixed $Q_{s}$ the fluid parameters should obey the relations $A_{s}=-\frac{1}{2} Q_{s}^{2}$ and hence $A_{s}$ are negative.

\subsection{The Hawking temperature}

The Hawking temperature of the black hole (3.5) (see also (3.15) ) may be calculated using the relation from [23]. It has the following form:

$$
T_{H}=\frac{d}{4 \pi(2 \mu)^{1 / d}} \prod_{s=1}^{m} H_{s 0}^{-h_{s}}
$$

where $H_{s 0}, s=1,2$, are defined in 3.13 .

For the dyonic solution from the previous subsection we get

$$
T_{H}=\frac{1}{8 \pi \mu}\left(H_{10} H_{20}\right)^{-1 / 2},
$$

where $T_{H}$ is a function of fluid parameters $A_{s}<0, s=1,2$. 


\section{Conclusions}

Here we have presented a family of spherically symmetric solutions with horizon in the model with multi-component anisotropic fluid with the equations of state (2.8) and the conditions (3.1) imposed. The metric of any solution contains $(n-1)$ Ricci-flat "internal" space metrics.

As in [6, 7, 8, the solutions are defined up to solutions of non-linear differential equations (equivalent to Todalike ones) with certain boundary conditions imposed. These solutions may have a polynomial structure when the matrix $A$ from (3.1) is coinciding with the Cartan matrix of some semi-simple finite-dimensional Lie algebra.

For certain equations of state (with $p_{i}= \pm \rho$ ) the metric of the solution may coincide with the metric of intersecting black branes (in a model with antisymmetric forms without dilatons). Here we have considered an example of simulating of $M 2-M 5$ black brane (dyonic) solution in $D=11$ supergravity with intersection rules corresponding to Lie algebra $A_{2}$.

An open problem is to generalize this formalism to the case when scalar fields are added into consideration. In a separate paper we also plan to calculate the post-Newtonian parameters $\beta$ and $\gamma$ corresponding to the 4dimensional section of the metric (for $d_{0}=2$ ) and analyze the thermodynamic properties of the black-brane-like solutions in the model with MCAF.

\section{Acknowledgments}

This work was supported in part by grant NPK-MU (PFUR) and Russian Foundation for Basic Research (Grant Nr. 09-02-00677-a.).

\section{References}

[1] K.A. Bronnikov, V.D. Ivashchuk and V.N. Melnikov Grav. Cosmol. 3, 203-212 (1997); gr-qc/9710054

[2] V.D Ivashchuk, V.N. Melnikov, J. Math. Phys. 39, 2866-2889 (1998); hep-th/9708157

[3] K.A. Bronnikov, Grav. Cosmol. 4, No 1 (13), 49 (1998); hep-th/9710207.

[4] V.D. Ivashchuk and V.N. Melnikov, In Lecture Notes in Physics, Vol. 537, "Mathematical and Quantum Aspects of Relativity and Cosmology Proceedings of the Second Samos Meeting on Cosmology, Geometry and Relativity held at Pythagoreon, Samos, Greece, 1998, eds: S. Cotsakis, G.W. Gibbons., Berlin, Springer, pp. 214-247, 2000; gr-qc/9901001.

[5] S. Cotsakis, V.D. Ivashchuk and V.N. Melnikov, Grav. Cosmol. 5, No 1 (17), $52-57$ (1999); gr-qc/9902148

[6] V.D. Ivashchuk and V.N. Melnikov, Grav. Cosmol. 5, No 4 (20), 313-318 (1999); gr-qc/0002085.

[7] V.D. Ivashchuk and V.N. Melnikov, Grav. Cosmol. 6, No 1 (21), 27-40 (2000); hep-th/9910041.

[8] V.D. Ivashchuk and V.N. Melnikov, Class. Quantum Grav. 17, 2073-2092 (2000); math-ph/0002048

[9] M.A. Grebeniuk, V.D. Ivashchuk and S.-W. Kim, J. Math. Phys. 43, 6016-6023 (2002); hep-th/0111219.

[10] M.A. Grebeniuk, V.D. Ivashchuk and V.N. Melnikov Phys. Lett. , B 543, 98-106 (2002); hep-th/0208083.

[11] V.D. Ivashchuk and V.N. Melnikov, Class. Quantum Grav. 18 R87-R152 (2001); hep-th/0110274

[12] V.D. Ivashchuk, V.N. Melnikov and A.B. Selivanov, Grav. Cosmol. 7 No. 4 (12), (2001); gr-qc/0205103.

[13] V.D. Ivashchuk, V.N. Melnikov and A.B. Selivanov, Grav. Cosmol. 9, No. 1-2 (33-34), 50-54 (2003); hep-th/0211247.

[14] H. Dehnen, V.D. Ivashchuk and V.N. Melnikov, Grav. Cosmol. 9, No. 3 (35), 153-158 (2003); gr-qc/0211049.

[15] H. Dehnen and V.D. Ivashchuk, J. Math. Phys. 45, 4726-4736 (2004); gr-qc/0310043.

[16] M. Cvetic and A. Tseytlin, Nucl. Phys. B 478, 181-198 (1996); hep-th/9606033

[17] I.Ya. Aref'eva, M.G. Ivanov and I.V. Volovich, Phys. Lett. B 406, 44-48 (1997); hep-th/9702079.

[18] N. Ohta, Phys. Lett. B 403, 218-224 (1997); hep-th/9702164

[19] V.D. Ivashchuk and S.-W. Kim, J. Math. Phys., 41 (1) 444-460 (2000); hep-th/9907019.

[20] V.D. Ivashchuk and V.N. Melnikov, Int. J. Mod. Phys. D 3, No. 4, 795-811 (1994); gr-qc/ 9403063

[21] V.R. Gavrilov, V.D. Ivashchuk, V.N. Melnikov, J. Math. Phys 36, 5829-5847 (1995).

[22] V.D. Ivashchuk, V.N. Melnikov and A.I. Zhuk, Nuovo Cim. B 104, No. 5, 575-581 (1989).

[23] J.W. York, Phys. Rev. D 31, 775 (1985).

[24] J. Fuchs and C. Schweigert, Symmetries, Lie algebras and Representations. A graduate course for physicists, Cambridge University Press, Cambridge, 1997.

[25] H. Lü, J. Maharana, S. Mukherji and C.N. Pope, Phys. Rev. D 57 2219-2229 (1997); hep-th/9707182.

[26] V.D. Ivashchuk, in preparation. 


\title{
On analogues of black brane solutions in the model with multicomponent anisotropic fluid
}

\author{
V. D. Ivashchuk ${ }^{1}$, \\ Center for Gravitation and Fundamental Metrology, VNIIMS, 46 Ozyornaya ul., Moscow 119361, Russia \\ Institute of Gravitation and Cosmology, Peoples' Friendship University of Russia, 6 Miklukho-Maklaya ul., \\ Moscow 117198, Russia
}

\begin{abstract}
A family of spherically symmetric solutions with horizon in the model with $m$-component anisotropic fluid is presented. The metrics are defined on a manifold that contains a product of $n-1$ Ricci-flat "internal" spaces. The equation of state for any $s$-th component is defined by a vector $U^{s}$ belonging to $\mathbb{R}^{n+1}$. The solutions are governed by moduli functions $H_{s}$ obeying non-linear differential equations with certain boundary conditions imposed. A simulation of black brane solutions in the model with antisymmetric forms is considered. An example of solution imitating $M_{2}-M_{5}$ configuration (in $D=11$ supergravity) corresponding to Lie algebra $A_{2}$ is presented.
\end{abstract}

\section{Introduction}

In this paper we continue our investigations of spherically-symmetric solutions with horizon (e.g., black brane ones) defined on product manifolds containing several Ricci-flat factor-spaces (with diverse signatures and dimensions). These solutions appear either in models with antisymmetric forms and scalar fields [1-[11] or in models with (multicomponent) anisotropic fluid [12]-[15]. For black brane solutions with 1-dimensional factor-spaces (of Euclidean signatures) see [16, 17, 18, and references therein.

These and more general brane cosmological and spherically symmetric solutions were obtained by reduction of the field equations to the Lagrange equations corresponding to Toda-like systems [2, 19]. An analogous reduction for models with multicomponent anisotropic fluids was performed earlier in [20, 21]. For cosmological-type models with antisymmetric forms without scalar fields any brane is equivalent to an anisotropic fluid with the equations of state:

$$
\hat{p}_{i}=-\hat{\rho} \quad \text { or } \quad \hat{p}_{i}=\hat{\rho},
$$

when the manifold $M_{i}$ belongs or does not belong to the brane worldvolume, respectively (here $\hat{p}_{i}$ is the effective pressure in $M_{i}$ and $\hat{\rho}$ is the effective density).

In this paper we present spherically-symmetric solutions with horizon (e.g the analogues of intersecting black brane solutions) in a model with multi-component anisotropic fluid (MCAF), when certain relations on fluid parameters are imposed. The solutions are governed by a set of moduli functions $H_{s}$ obeying non-linear differential master equations with certain boundary conditions imposed. These master equations are equaivalent to Toda-like equations and depend upon the non-degenerate $(m \times m)$ matrix $A$. It was conjectured earlier that the functions $H_{s}$ should be polynomials when $A$ is a Cartan matrix for some semisimple finite-dimensional Lie algebra (of rank m) [6]. This conjecture was verified for Lie algebras: $A_{m}, C_{m+1}, m \geq 1$ [7, 8]. A special case of black hole solutions with MCAF corresponding to semisimple Lie algebra $A_{1} \oplus \ldots \oplus A_{1}$ was considered earlier in [13] (for $m=1$ see [12]).

The paper is organized as follows. In Section 2 the model is formulated. In Section 3 spherically-symmetric MCAF solutions with horizon corresponding to black brane type solutions, are presented. In Section 4 a polynomial structure of moduli functions $H_{s}$ for semisimple finite-dimensional Lie algebras is discussed. In Section 5 a simulation of intersecting black brane solutions is considered and an analogue of $M 2-M 5$ dyonic solution is presented.

\section{The model}

In this paper we deal with a family of spherically symmetric solutions to Einstein equations with an anisotropic matter source

$$
R_{N}^{M}-\frac{1}{2} \delta_{N}^{M} R=k^{2} T_{N}^{M},
$$

\footnotetext{
${ }^{1}$ e-mail: ivashchuk@mail.ru
} 
defined on the manifold

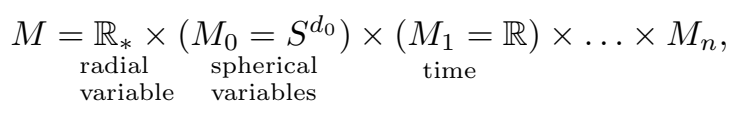

with the block-diagonal metrics

$$
d s^{2}=\mathrm{e}^{2 \gamma(u)} d u^{2}+\sum_{i=0}^{n} \mathrm{e}^{2 \beta^{i}(u)} h_{m_{i} n_{i}}^{[i]} d y^{m_{i}} d y^{n_{i}} .
$$

Here $\mathbb{R}_{*} \subseteq \mathbb{R}$ is an open interval. The manifold $M_{i}$ with the metric $h^{[i]}, i=1,2, \ldots, n$, is a Ricci-flat space of dimension $d_{i}$ :

$$
R_{m_{i} n_{i}}\left[h^{[i]}\right]=0,
$$

and $h^{[0]}$ is the standard metric on the unit sphere $S^{d_{0}}$, so that

$$
R_{m_{0} n_{0}}\left[h^{[0]}\right]=\left(d_{0}-1\right) h_{m_{0} n_{0}}^{[0]}
$$

$u$ is a radial variable, $\kappa^{2}$ is the gravitational constant, $d_{1}=1$ and $h^{[1]}=-d t \otimes d t$.

The energy-momentum tensor is adopted in the following form for each component of the fluid:

$$
\left(T_{N}^{s M}\right)=\operatorname{diag}\left(-\hat{\rho}^{s}, \hat{p}_{0}^{s} \delta_{k_{0}}^{m_{0}}, \hat{p}_{1}^{s} \delta_{k_{1}}^{m_{1}}, \ldots, \hat{p}_{n}^{s} \delta_{k_{n}}^{m_{n}}\right),
$$

where $\hat{\rho}^{s}$ and $\hat{p}_{i}^{s}$ are the effective density and pressures respectively, depending on the radial variable $u$.

We assume that the following "conservation laws"

$$
\nabla_{M} T_{N}^{(s) M}=0
$$

are valid for all components.

We also impose the following equations of state

$$
\hat{p}_{i}^{s}=\left(1-\frac{2 U_{i}^{s}}{d_{i}}\right) \hat{\rho}^{s}
$$

where $U_{i}^{s}$ are constants, $i=0,1, \ldots, n$.

The physical density and pressures are related to the effective ones (with "hats") by the formulae

$$
\rho^{s}=-\hat{p}_{1}^{s}, \quad p_{u}^{s}=-\hat{\rho}^{s}, \quad p_{i}^{s}=\hat{p}_{i}^{s} \quad(i \neq 1) .
$$

In what follows we put $\kappa=1$ for simplicity.

\section{Spherically symmetric solutions with horizon}

We will make the following assumptions:

$$
\begin{array}{ll}
1^{o} . & U_{0}^{s}=0 \Leftrightarrow \hat{p}_{0}^{s}=\hat{\rho}^{s}, \\
2^{o} . & U_{1}^{s}=1 \Leftrightarrow \hat{p}_{1}^{s}=-\hat{\rho}^{s}, \\
3^{o} . & \left(U^{s}, U^{s}\right)=U_{i}^{s} G^{i j} U_{j}^{s}>0, \\
4^{o} . & 2\left(U^{s}, U^{l}\right) /\left(U^{l}, U^{l}\right)=A_{s l},
\end{array}
$$

where $A=\left(A_{s l}\right)$ is non-degenerate matrix,

$$
G^{i j}=\frac{\delta^{i j}}{d_{i}}+\frac{1}{2-D}
$$

are components of the matrix inverse to the matrix of the minisuperspace metric 22$]$

$$
\begin{aligned}
& \left(G_{i j}\right)=\left(d_{i} \delta_{i j}-d_{i} d_{j}\right) \\
& i, j=0,1, \ldots, n \text { and } D=1+\sum_{i=0}^{n} d_{i} \text { is the total dimension. }
\end{aligned}
$$


The conditions $1^{\circ}$ and $2^{\circ}$ in brane terms mean that brane "lives" in the time manifold $M_{1}$ and does not "live" in $M_{0}$. Due to assumptions $1^{\circ}$ and $2^{\circ}$ and the equations of state (2.8), the energy-momentum tensor (2.6) reads as follows:

$$
\left(T_{N}^{(s)} \begin{array}{l}
M \\
N
\end{array}\right)=\operatorname{diag}\left(-\rho^{s}, \rho^{s} \delta_{k_{0}}^{m_{0}},-\rho^{s}, p_{2}^{s} \delta_{k_{2}}^{m_{2}}, \ldots, p_{n}^{s} \delta_{k_{n}}^{m_{n}}\right)
$$

Under the conditions (2.8) and (3.1) we have obtained the following black-brane-like solutions to the HilbertEinstein equations (2.1):

$$
\begin{aligned}
& d s^{2}=J_{0}\left(\frac{d R^{2}}{1-2 \mu / R^{d}}+R^{2} d \Omega_{d_{0}}^{2}\right)-J_{1}\left(1-\frac{2 \mu}{R^{d}}\right) d t^{2}+\sum_{i=2}^{n} J_{i} h_{m_{i} n_{i}}^{[i]} d y^{m_{i}} d y^{n_{i}}, \\
& \rho^{s}=-\frac{A_{s}}{J_{0} R^{2 d_{0}}} \prod_{l=1}^{m} H_{l}^{-A_{s l}},
\end{aligned}
$$

which may be derived by analogy with the black brane solutions [7, 8]. Here $d=d_{0}-1$,

$$
d \Omega_{d_{0}}^{2}=h_{m_{0} n_{0}}^{[0]} d y^{m_{0}} d y^{n_{0}}
$$

is the $d_{0}$-dimensional spherical element (corresponding to the metric on $S^{d_{0}}$ ),

$$
J_{i}=\prod_{s=1}^{m} H_{s}^{-2 h_{s} U^{s i}}
$$

$i=0,1, \ldots, n, \mu>0$ is integration constant and

$$
\begin{aligned}
& U^{s i}=G^{i j} U_{j}^{s}=\frac{U_{i}^{s}}{d_{i}}+\frac{1}{2-D} \sum_{j=0}^{n} U_{j}^{s}, \\
& h_{s}=K_{s}^{-1}, \quad K_{s}=\left(U^{s}, U^{s}\right) .
\end{aligned}
$$

It follows from $1^{o}$ and 3.9 that

$$
U^{s 0}=\frac{1}{2-D} \sum_{j=1}^{n} U_{j}^{s}
$$

Functions $H_{s}>0$ obey the equations

$$
R^{d_{0}} \frac{d}{d R}\left[\left(1-\frac{2 \mu}{R^{d}}\right) \frac{R^{d_{0}}}{H_{s}} \frac{d H_{s}}{d R}\right]=B_{s} \prod_{l=1}^{m} H_{l}^{-A_{s l}},
$$

with $B_{s}=2 K_{s} A_{s}$ and the boundary conditions imposed:

$$
H_{s} \rightarrow H_{s 0} \neq 0, \text { for } \quad R^{d} \rightarrow 2 \mu,
$$

and

$$
H_{s}(R=+\infty)=1,
$$

$s=1, \ldots, m$.

Here we also impose the following (additional) condition on the solutions

$$
H_{s}(R)>0 \text { is smooth in }\left(R_{\epsilon},+\infty\right) \text {, }
$$

$s=1, \ldots, m$, where $R_{\epsilon}=(2 \mu)^{1 / d} e^{-\epsilon}, \epsilon>0$. Then the metric (3.5) has a regular horizon at $R^{d}=2 \mu$ and has an asymptotically flat $\left(2+d_{0}\right)$-dimensional section.

Due to to (3.1) and (3.9) the metric reads

$$
d s^{2}=J_{0}\left[\frac{d R^{2}}{1-2 \mu / R^{d}}+R^{2} d \Omega_{d_{0}}^{2}-\left(\prod_{s=1}^{m} H_{s}^{-2 h_{s}}\right)\left(1-\frac{2 \mu}{R^{d}}\right) d t^{2}+\sum_{i=2}^{n} Y_{i} h_{m_{i} n_{i}}^{[i]} d y^{m_{i}} d y^{n_{i}}\right],
$$

where

$$
Y_{i}=\prod_{s=1}^{m} H_{s}^{-2 h_{s} U_{i}^{s} / d_{i}} .
$$

The solution (3.5), 3.6 may be verified just by a straightforward substitution into equations of motion. A detailed derivation of this solution will be given in a separate paper [27. A special orthogonal case when $\left(U^{s}, U^{l}\right)=0$, for $s \neq l$, was considered earlier in [13] (for $m=1$ see [12]) More general solutions in orthogonal case (with more general condition instead of $2^{\circ}$ ) were obtained in [15] (for $m=1$ see [14.) 


\section{Polynomial structure of $H_{s}$ for Lie algebras}

Now we deal with solutions to second order non-linear differential equations (3.12) that may be rewritten as follows

$$
\frac{d}{d z}\left(\frac{(1-2 \mu z)}{H_{s}} \frac{d}{d z} H_{s}\right)=\bar{B}_{s} \prod_{l=1}^{m} H_{l}^{-A_{s l}}
$$

where $H_{s}(z)>0, z=R^{-d} \in\left(0,(2 \mu)^{-1}\right)(\mu>0)$ and $\bar{B}_{s}=B_{s} / d^{2} \neq 0$. Eqs. (3.13) and (3.14) read

$$
\begin{array}{r}
H_{s}\left((2 \mu)^{-1}-0\right)=H_{s 0} \in(0,+\infty), \\
H_{s}(+0)=1,
\end{array}
$$

$s=1, \ldots, m$.

The condition (3.15) reads as follows

$$
H_{s}(z)>0 \text { is smooth in }\left(0, z_{\epsilon}\right),
$$

$s=1, \ldots, m$, where $z_{\epsilon}=(2 \mu)^{-1} e^{\epsilon d}, \epsilon>0$.

It was conjectured in [6] that equations (4.1)-(4.3) have polynomial solutions when $\left(A_{s s^{\prime}}\right)$ is a Cartan matrix for some semisimple finite-dimensional Lie algebra $\mathcal{G}$ of rank $m$. In this case we get

$$
H_{s}(z)=1+\sum_{k=1}^{n_{s}} P_{s}^{(k)} z^{k}
$$

where $P_{s}^{(k)}$ are constants, $k=1, \ldots, n_{s} ; P_{s}^{\left(n_{s}\right)} \neq 0$, and

$$
n_{s}=b_{s} \equiv 2 \sum_{l=1}^{m} A^{s l}
$$

$s=1, \ldots, m$, are the components of twice the dual Weyl vector in the basis of simple co-roots 24 . Here $\left(A^{s l}\right)=$ $\left(A_{s l}^{-1}\right)$.

This conjecture was verified for $\mathbf{A}_{\mathbf{m}}$ and $\mathbf{C}_{\mathbf{m}+\mathbf{1}}$ series of Lie algebras in [7, 8, In the extremal case $(\mu=+0)$ an analogue of this conjecture was suggested (implicitly) in [25].

Remark. We note that the substitution of (2.6), (2.8), (3.5), (3.6) into Hilbert-Einstein equations (2.1) gives an extra equation

$$
E_{T}=\frac{d^{2}}{4} \sum_{s, l=1}^{m} h_{s} A_{s l}\left[F \frac{d}{d z} \ln H_{s}+\mu b_{s}\right]\left[F \frac{d}{d z} \ln H_{l}+\mu b_{l}\right]+\sum_{s=1}^{m} A_{s} F \prod_{l=1}^{m} H_{l}^{-A_{s l}}=\frac{1}{2} \sum_{s=1}^{m} h_{s} b_{s}(\mu d)^{2},
$$

where $F=1-2 \mu z . E_{T}$ is an integral of motion for the set of equations (4.1). The constraint (4.7) is satisfied identically due to (4.2), (4.4) (one can check this by putting $2 \mu z=1$ ).

$\mathbf{A}_{1} \oplus \ldots \oplus \mathbf{A}_{1}$-case.

The simplest example occurs in the orthogonal case : $\left(U^{s}, U^{l}\right)=0$, for $s \neq l$ 1, 2] (see also [16, 17, 18, and refs. therein). In this case $\left(A_{s l}\right)=\operatorname{diag}(2, \ldots, 2)$ is a Cartan matrix for the semisimple Lie algebra $\mathbf{A}_{\mathbf{1}} \oplus \ldots \oplus \mathbf{A}_{\mathbf{1}}$ and

$$
H_{s}(z)=1+P_{s} z
$$

with $P_{s} \neq 0$, satisfying

$$
P_{s}\left(P_{s}+2 \mu\right)=-\bar{B}_{s}=-2 K_{s} A_{s} / d^{2},
$$

$s=1, \ldots, m$. When all $A_{s}<0$ (or, equivalently, $\rho^{s}>0$ ) there exists a unique set of numbers $P_{s}>0$ obeying (4.9).

$A_{2}$-case.

For the Lie algebra $\mathcal{G}$ coinciding with $\mathbf{A}_{\mathbf{2}}=\operatorname{sl}(3)$ we get $n_{1}=n_{2}=2$ and

$$
H_{s}=1+P_{s} z+P_{s}^{(2)} z^{2},
$$

where $P_{s}=P_{s}^{(1)}$ and $P_{s}^{(2)} \neq 0$ are constants, $s=1,2$. 
It was found in $[6]$ that for $P_{1}+P_{2}+4 \mu \neq 0$ (e.g. when all $P_{s}>0$ ) the following relations take place

$$
P_{s}^{(2)}=\frac{P_{s} P_{s+1}\left(P_{s}+2 \mu\right)}{2\left(P_{1}+P_{2}+4 \mu\right)}, \quad \bar{B}_{s}=-\frac{P_{s}\left(P_{s}+2 \mu\right)\left(P_{s}+4 \mu\right)}{P_{1}+P_{2}+4 \mu},
$$

$s=1,2$.

Here we denote $s+1=2,1$ for $s=1,2$, respectively.

Other solutions.

At the moment the "master" equations were integrated (using Maple) in 9, 10, for Lie algebras $\mathbf{C}_{\mathbf{2}}$ and $\mathbf{A}_{\mathbf{3}}$, respectively. (For $\mathbf{D}_{4}$-polynomials in the extremal case $\mu \rightarrow+0$ see [25].)

Special solutions $H_{s}(z)=\left(1+P_{s} z\right)^{b_{s}}$ with $b_{s}$ from (4.6) appeared earlier in 3, 4, 5, in a context of so-called block-orthogonal configurations.

Extremal case. For $\mu \rightarrow+0$ the conditions (4.2), (4.4) should be omitted but we should impose the relation

$$
E_{T}=\frac{d^{2}}{4} \sum_{s, l=1}^{m} h_{s} A_{s l} \frac{d}{d z} \ln H_{s} \frac{d}{d z} \ln H_{l}+\sum_{s=1}^{m} A_{s} F \prod_{l=1}^{m} H_{l}^{-A_{s l}}=0,
$$

following from (4.7). The functions $H_{s}(z)>0$ obeying (4.1) (with $\left.\mu \rightarrow+0\right)$ are smooth on $(0,+\infty)$. For certain relations on $U^{s}$-vectors imposed the solution under consideration has a horizon for $R \rightarrow+0(z \rightarrow+\infty)$, e.g. it may describe analogues of extremal black brane solutions [11.

When the boundary condition (4.3) is omitted we get a special solution with

$$
H_{s}(z)=C_{s} z^{b_{s}},
$$

and

$$
C_{s}=\prod_{l=1}^{m}\left(-b_{l} / \bar{B}_{l}\right)^{-A^{s l}}
$$

where $b_{s} / \bar{B}_{s}<0, s=1, \ldots, m$. The metric (3.5) with $H_{s}$ from (4.13) has no an asymptotically flat $\left(2+d_{0}\right)$ dimensional section.

It should be noted that the solutions obeying $H_{s}(+0)=1$ and $b_{s} / \bar{B}_{s}<0$ have an asymptotical behaviour $H_{s}(z) \sim C_{s} z^{b_{s}}$ for $z \rightarrow+\infty$ (e.g. in the near-horizon limit $R \rightarrow+0$ of extremal black-brane-type solutions).

\section{$5 \quad$ Examples}

\subsection{Analogues of intersecting black brane solutions}

The solution from the previous section for MCAF allows one to simulate the intersecting black brane solutions [11] in the model with antisymmetric forms without scalar fields. In this case the parameters $U_{i}^{s}$ and pressures have the following form:

$$
\begin{aligned}
U_{i}^{s}=\begin{array}{c}
d_{i}, \\
0,
\end{array} \quad p_{i}^{s}=-\rho^{s}, & i \in I_{s} ; \\
\rho^{s}, & i \notin I_{s} .
\end{aligned}
$$

Here $I_{s}=\left\{i_{1}^{s}, \ldots, i_{k_{s}}^{s}\right\} \in\{1, \ldots n\}$ is the index set [11] corresponding to brane submanifold $M_{i_{1}^{s}} \times \ldots \times M_{i_{k_{s}}^{s}}$.

The relation $4^{o}$ (3.1) leads us to the following dimensions of intersections of brane submanifolds ("worldvolumes") [2, 11]:

$$
d\left(I_{s} \cap I_{l}\right)=\frac{d\left(I_{s}\right) d\left(I_{l}\right)}{D-2}+\frac{1}{2} K_{l} A_{s l},
$$

$s \neq l ; s, l=1, \ldots, m$. Here $d\left(I_{s}\right)$ and $d\left(I_{l}\right)$ are dimensions of brane worldvolumes. 


\section{$5.2 \quad M_{2}-M_{5}$-analogue for Lie algebra $A_{2}$}

In [13] examples of MCAF-analogues of $M 2 \cap M 5, M 2 \cap M 2, M 5 \cap M 5$ black brane solutions in $D=11$ supergravity, with the standard (orthogonal) intersection rules were considered.

Now we consider a solution with 2-component anisotropic fluid that simulates $M_{2}-M_{5}$ dyonic configuration in $D=11$ supergravity [6], corresponding to Lie algebra $\mathbf{A}_{\mathbf{2}}$.

The solution is defined on the manifold

$$
M=(2 \mu,+\infty) \times\left(M_{0}=S^{2}\right) \times\left(M_{1}=\mathbb{R}\right) \times M_{2} \times M_{3},
$$

where $\operatorname{dim} M_{2}=2$ and $\operatorname{dim} M_{3}=5$. The $U^{s}$-vectors corresponding to fluid components obey (5.1) with $I_{1}=\{1,2\}$ and $I_{2}=\{1,3\}$.

The solution reads as following

$$
\begin{array}{r}
g=H_{1}^{1 / 3} H_{2}^{2 / 3}\left\{\frac{d R \otimes d R}{1-2 \mu / R}+R^{2} h\left[S^{2}\right]-H_{1}^{-1} H_{2}^{-1}\left(1-\frac{2 \mu}{R}\right) d t \otimes d t+H_{1}^{-1} h^{[2]}+H_{2}^{-1} h^{[3]}\right\}, \\
\rho^{1}=-\frac{A_{1}}{J_{0} R^{4}} H_{1}^{-2} H_{2}, \quad \rho^{2}=-\frac{A_{2}}{J_{0} R^{4}} H_{1} H_{2}^{-2},
\end{array}
$$

where $J_{0}=H_{1}^{1 / 3} H_{2}^{2 / 3} ; h\left[S^{2}\right]$ is the canonical metric on 2-dimensional sphere $S^{2}, h^{[2]}$ and $h^{[3]}$ are Ricci-flat metrics of Euclidean signatures defined on the manifolds $M_{2}$ and $M_{3}$, respectively; $\mu>0$ and $H_{s}$ are defined by (4.10), where $z=R^{-1}$ and parameters $P_{s}, P_{s}^{(2)}, \bar{B}_{s}=B_{s}=4 A_{s}(s=1,2)$ obey (4.11).

This solution simulates $\mathbf{A}_{\mathbf{2}}$-dyon from [6] consisting of an electric $M 2$-brane with a worldvolume isomorphic to $\left(M_{1}=\mathbb{R}\right) \times M_{2}$ and a magnetic $M 5$-brane with a worldvolume isomorphic to $\left(M_{1}=\mathbb{R}\right) \times M_{3}$. The branes are intersecting on the time manifold $M_{1}=\mathbb{R}$. Here $K_{s}=\left(U^{s}, U^{s}\right)=2$, for all $s=1,2$.

For the $\mathbf{A}_{2}$-dyon from [6] we had $\bar{B}_{s}=B_{s}=-2 Q_{s}^{2}$, where $Q_{s}$ is the charge density parameter of $s$-th brane. Thus, for fixed $Q_{s}$ the fluid parameters should obey the relations $A_{s}=-\frac{1}{2} Q_{s}^{2}$ and hence $A_{s}$ are negative.

Let us consider the extremal case $\mu \rightarrow+0$ of the solution (5.4)-(5.5) with $A_{1}<0$ and $A_{2}<0$. The nearhorizon limit $(R \rightarrow+0)$ gives us an exact solution (see (4.13))

$$
\begin{array}{r}
g=C_{1}^{1 / 3} C_{2}^{2 / 3}\left\{h\left[A d S^{2}\right]+h\left[S^{2}\right]+C_{1}^{-1} h^{[2]}+C_{2}^{-1} h^{[3]}\right\}, \\
\rho^{1}=-A_{1} C_{1}^{-7 / 3} C_{2}^{1 / 3}, \quad \rho^{2}=-A_{2} C_{1}^{2 / 3} C_{2}^{-8 / 3},
\end{array}
$$

where $C_{1}=2\left|A_{1}\right|^{2 / 3}\left|A_{2}\right|^{1 / 3}$ and $C_{2}=2\left|A_{2}\right|^{2 / 3}\left|A_{1}\right|^{1 / 3}, h\left[A d S^{2}\right]=R^{-2}\left(d R \otimes d R-R^{4} d \bar{t} \otimes d \bar{t}\right)$ is the metric of (the half of) the anti-deSitter space $A d S^{2}$ (here $\bar{t}=C_{1}^{-1 / 2} C_{2}^{-1 / 2} t$ ). Thus, we have obtained a static configuration defined on (the half of) the product space $A d S^{2} \times S^{2} \times M_{2} \times M_{3}$. (For the solution with two branes and $C_{1}=C_{2}=1$ see [26.)

Analogues of $M 2, M 5$ and $D 3$ solutions. Here we outline for completeness the analogues of non-marginal M2, M5 $(D=11)$ and $D 3(D=10)$ black brane solutions. The solutions are defined on the product manifolds $M=(2 \mu,+\infty) \times\left(M_{0}=S^{d_{0}}\right) \times\left(M_{1}=\mathbb{R}\right) \times M_{2}$, where $\operatorname{dim} M_{2}=d_{2}=2,5,3$ and $d_{0}=7,4,5$ for $M 2, M 5, D 3$ branes, respectively. The vector $U=U^{1}$ has the components: $U_{0}=0$ and $U_{i}=1$ for $i>0$. The solutions read as follows

$$
\begin{array}{r}
g=H^{r}\left\{\frac{d R \otimes d R}{1-2 \mu R^{-d}}+R^{2} h\left[S^{d_{0}}\right]-H^{-1}\left(1-2 \mu R^{-d}\right) d t \otimes d t+H^{-1} h^{[2]}\right\}, \\
\rho=\rho^{1}=-A H^{-2-r} R^{-2 d_{0}},
\end{array}
$$

where $h^{[2]}$ is a Ricci-flat metric on $M_{2}, h\left[S^{d_{0}}\right]$ is the canonical metric on $d_{0}$-dimensional sphere $S^{d_{0}}, H=$ $1+P R^{-d}, P(P+2 \mu)=-4 A / d^{2}(A<0, P>0, \mu>0), d=d_{0}-1$ and $r=1 / 3,2 / 3,1 / 2$ for $M 2, M 5, D 3$ branes, respectively. In the extremal case $\mu \rightarrow+0$ the near-horizon limit gives an exact solution for the flat space $\left(M_{2}=\mathbb{R}^{d_{2}}, h^{[2]}\right)$

$$
\begin{array}{r}
g=P^{r}\left\{h\left[S^{d_{0}}\right]+\frac{4}{(d-2)^{2}} h\left[A d S^{d_{2}+2}\right]\right\}, \\
\rho=-A P^{-2-r}, \quad P^{2}=-4 A / d^{2},
\end{array}
$$

where $h\left[A d S^{k+2}\right]=d u \otimes d u+e^{2 u}\left(-d y^{0} \otimes d y^{0}+\sum_{i=1}^{k} d y^{i} \otimes d y^{i}\right)$ is the metric of (the part of) the anti-deSitter space $A d S^{k+2}$. Thus, we are led to a static configurations defined on (the parts of) the product spaces $S^{d_{0}} \times A d S^{d_{2}+2}$, i.e. $S^{7} \times A d S^{4}, S^{4} \times A d S^{7}$ and $S^{5} \times A d S^{5}$ for $M 2, M 5$ and $D 3$ branes, respectively. It should be pointed out that the solutions with $A d S^{k}$ factor spaces may be of interest due to possible application in a context of $A d S / C F T$ approach [28, 29, 30] (or its modifications). 


\subsection{The Hawking temperature}

The Hawking temperature of the black hole (3.5) (see also (3.16)) may be calculated using the relation from [23]. It has the following form:

$$
T_{H}=\frac{d}{4 \pi(2 \mu)^{1 / d}} \prod_{s=1}^{m} H_{s 0}^{-h_{s}},
$$

where $H_{s 0}, s=1,2$, are defined in (3.13).

For the dyonic solution from the previous subsection we get

$$
T_{H}=\frac{1}{8 \pi \mu}\left(H_{10} H_{20}\right)^{-1 / 2},
$$

where $T_{H}$ is a function of fluid parameters $A_{s}<0, s=1,2$.

\section{Conclusions}

Here we have presented a family of spherically symmetric solutions with horizon in the model with multi-component anisotropic fluid with the equations of state (2.8) and the conditions (3.1) imposed. The metric of any solution contains $(n-1)$ Ricci-flat "internal" space metrics.

As in $6,7,8$, the solutions are defined up to solutions of non-linear differential equations (equivalent to Todalike ones) with certain boundary conditions imposed. These solutions may have a polynomial structure when the matrix $A$ from (3.1) is coinciding with the Cartan matrix of some semi-simple finite-dimensional Lie algebra.

For certain equations of state (with $p_{i}= \pm \rho$ ) the metric of the solution may coincide with the metric of intersecting black branes (in a model with antisymmetric forms without dilatons). Here we have considered an example of simulating of $M 2-M 5$ black brane (dyonic) solution in $D=11$ supergravity with intersection rules corresponding to the Lie algebra $A_{2}$. We have also outlined the analogues of non-marginal $M 2, M 5$ and D3 black brane solutions. In the extremal case $\mu \rightarrow+0$ the near-horizon limits of all these solutions were found.

An open problem is to generalize this formalism to the case when scalar fields are added into consideration. In a separate paper we also plan to calculate the post-Newtonian parameters $\beta$ and $\gamma$ corresponding to the 4dimensional section of the metric (for $d_{0}=2$ ) and analyze the thermodynamic properties of the black-brane-like solutions in the model with MCAF.

\section{Acknowledgments}

This work was supported in part by grant NPK-MU (PFUR) and Russian Foundation for Basic Research (Grant Nr. 09-02-00677-a.).

\section{References}

[1] K.A. Bronnikov, V.D. Ivashchuk and V.N. Melnikov Grav. Cosmol. 3, 203-212 (1997); gr-qc/9710054

[2] V.D Ivashchuk, V.N. Melnikov, J. Math. Phys. 39, 2866-2889 (1998); hep-th/9708157

[3] K.A. Bronnikov, Grav. Cosmol. 4, No 1 (13), 49 (1998); hep-th/9710207.

[4] V.D. Ivashchuk and V.N. Melnikov, In Lecture Notes in Physics, Vol. 537, "Mathematical and Quantum Aspects of Relativity and Cosmology Proceedings of the Second Samos Meeting on Cosmology, Geometry and Relativity held at Pythagoreon, Samos, Greece, 1998, eds: S. Cotsakis, G.W. Gibbons., Berlin, Springer, pp. 214-247, 2000; gr-qc/9901001.

[5] S. Cotsakis, V.D. Ivashchuk and V.N. Melnikov, Grav. Cosmol. 5, No 1 (17), $52-57$ (1999); gr-qc/9902148

[6] V.D. Ivashchuk and V.N. Melnikov, Grav. Cosmol. 5, No 4 (20), 313-318 (1999); gr-qc/0002085.

[7] V.D. Ivashchuk and V.N. Melnikov, Grav. Cosmol. 6, No 1 (21), 27-40 (2000); hep-th/9910041.

[8] V.D. Ivashchuk and V.N. Melnikov, Class. Quantum Grav. 17, 2073-2092 (2000); math-ph/0002048

[9] M.A. Grebeniuk, V.D. Ivashchuk and S.-W. Kim, J. Math. Phys. 43, 6016-6023 (2002); hep-th/0111219

[10] M.A. Grebeniuk, V.D. Ivashchuk and V.N. Melnikov Phys. Lett. , B 543, 98-106 (2002); hep-th/0208083.

[11] V.D. Ivashchuk and V.N. Melnikov, Class. Quantum Grav. 18 R87-R152 (2001); hep-th/0110274

[12] V.D. Ivashchuk, V.N. Melnikov and A.B. Selivanov, Grav. Cosmol. 7 No. 4 (12), (2001); gr-qc/0205103.

[13] V.D. Ivashchuk, V.N. Melnikov and A.B. Selivanov, Grav. Cosmol. 9, No. 1-2 (33-34), 50-54 (2003); hep-th/0211247

[14] H. Dehnen, V.D. Ivashchuk and V.N. Melnikov, Grav. Cosmol. 9, No. 3 (35), 153-158 (2003); gr-qc/0211049. 
[15] H. Dehnen and V.D. Ivashchuk, J. Math. Phys. 45, 4726-4736 (2004); gr-qc/0310043.

[16] M. Cvetic and A. Tseytlin, Nucl. Phys. B 478, 181-198 (1996); hep-th/9606033.

[17] I.Ya. Aref'eva, M.G. Ivanov and I.V. Volovich, Phys. Lett. B 406, 44-48 (1997); hep-th/9702079.

[18] N. Ohta, Phys. Lett. B 403, 218-224 (1997); hep-th/9702164

[19] V.D. Ivashchuk and S.-W. Kim, J. Math. Phys., 41 (1) 444-460 (2000); hep-th/9907019.

[20] V.D. Ivashchuk and V.N. Melnikov, Int. J. Mod. Phys. D 3, No. 4, 795-811 (1994); gr-qc/ 9403063

[21] V.R. Gavrilov, V.D. Ivashchuk, V.N. Melnikov, J. Math. Phys 36, 5829-5847 (1995).

[22] V.D. Ivashchuk, V.N. Melnikov and A.I. Zhuk, Nuovo Cim. B 104, No. 5, 575-581 (1989).

[23] J.W. York, Phys. Rev. D 31, 775 (1985).

[24] J. Fuchs and C. Schweigert, Symmetries, Lie algebras and Representations. A graduate course for physicists, Cambridge University Press, Cambridge, 1997.

[25] H. Lü, J. Maharana, S. Mukherji and C.N. Pope, Phys. Rev. D 57 2219-2229 (1997); hep-th/9707182

[26] V.D. Ivashchuk, Phys. Lett., B 434, No 1, 28-35 (1998); hep-th/9704113.

[27] V.D. Ivashchuk, in preparation.

[28] J.M. Maldacena, Adv. in Theor. Math. Phys. 2: 231252 (1998); hep-th/9711200

[29] S.S. Gubser, I.R. Klebanov and A.M. Polyakov, Phys. Lett. B 428, 105114 (1998); hep-th/9802109.

[30] E. Witten (1998), Adv. in Theor. Math. Phys. 2, 253291 (1998); hep-th/9802150. 